Voix et Images

voixetimages

\title{
Hubert Aquin et le jeu de l'écriture
}

\section{Anne Gagnon}

Volume 1, numéro 1, septembre 1975

\section{Hubert Aquin}

URI : https://id.erudit.org/iderudit/013981ar

DOI : https://doi.org/10.7202/013981ar

Aller au sommaire du numéro

Éditeur(s)

Les Presses de l'Université du Québec

ISSN

0318-9201 (imprimé)

1705-933X (numérique)

Découvrir la revue

Citer ce document

Gagnon, A. (1975). Hubert Aquin et le jeu de l'écriture. Voix et Images, 1(1),

5-18. https://doi.org/10.7202/013981ar d'utilisation que vous pouvez consulter en ligne.

https://apropos.erudit.org/fr/usagers/politique-dutilisation/ 


\section{Hubert Aquin et le jeu de l'écriture}

L'entrevue que nous présentons à nos lecteurs est extraite d'un ouvrage collectif, à paraître bientôt, préparé sous la direction d'Anne Gagnon, professeur au département d'Études litterraires de l'Université du Québec à Montréal. Après avoir analysé l'œuvre de H. Aquin, A. Gagnon a voulu mettre en rapport le discours critique et la parole vive de l'écrivain.

\section{LE RAPPORT ÉCRIVAIN-ÉCRITURE}

A. G. Une phrase de Roland Barthes dans Critique et vérité me semble bien traduire votre rapport, complexe, à l'écriture. II parle de la méthode et dit qu'elle est "l'acte de doute par lequel on s'interroge sur le hasard ou la nature" ( $p$. 15). Une méthode ne serait pas seulement ce qu'un lecteur utilise pour se retrouver dans un livre et le réorganiser mais il y aurait un style d'écriture, qui est le rapport de l'auteur à sa propre narration, ce rapport n'allant pas de soi. Barthes ajoute: "Écrire ce n'est pas engager un rapport facile avec une moyenne de tous les lecteurs possibles, mais c'est engager un rapport difficile avec notre propre langage. " ( $p .34)$

H. A. Je trouve que cette conception est tournée vers soi; l'explication de Barthes est solipsiste dans la mesure où la dialectique est à l'intérieur de la réflexivité, de l'écrivain avec son écriture ou son langage. C'est un rapport entre lui et son propre langage, qu'il manipule parce qu'il l'a hérité culturellement.

A. G. Au contraire, parce qu'alors il s'agirait de cette moyenne de lecteurs. Barthes fait une difference entre écrivains et écrivants. Ces derniers utilisent le langage dans sa fonction instrumentale alors que les écrivains font un travail dans le langage, énonçant les conditions même de la narration et pour eux l'écriture fait problème. N'est-ce pas ce qui se passe dans vos romans?

H. A. Oui, mais ce qui me dérange un peu dans les deux catégories de Barthes, c'est que cela confine l'écrivain dans une position terriblement intense. Je dis que l'écrivain peut fort bien se permettre d'être 
écrivain et écrivant en même temps ou alternativement. Que l'écrivain doive toujours être au sommet du langage, dans une situation de tension avec le langage, ce n'est pas ce que je resseils.

A. G. Ce que vous appelez un rapport d'intensité est peut-être tout simplement un rapport de Vérité.

H. A: Ce qui est un rapport extrêmement crispant et stressant parce que je ne vois pas pourquoi je serais mandaté pour exprimer cette vérité ontologique par des livres ou dans des livres.

A. G. Mais la vérité ce n'est pas quelque chose d'extérieur à vous, c'est ce que vous êtes et exprimez dans vos livres. Même les jeux de cachecache, les leurres, etc. Vos romans sont une des formes de votre réalité.

H. A. Alors je suis d'accord avec vous mais pas avec la catégorie du degré zéro de Barthes car j'irais jusqu'à dire que le Code civil, les volumes de jurisprudence, c'est de la littérature. C'est une production littéraire phénoménale. Et les traités scientifiques du xvıe siècle. Aujourd'hui, on peut les considérer comme tels et les analyser d'un point de vue esthétique. Je pense la même chose des traités scientifiques contemporains même si cela peut choquer les physiciens nucléaires. Car dans deux siècles ils auront la même valeur que ceux du $x v \|^{\circledR}$ siècle actuellement. Le degré zéro est donc idéal. Je trouve qu'un traité scientifique pourrait être intégré à la limite à un inventaire littéraire d'un siècle, de même que la jurisprudence et une bonne partie des actes notariés.

A. G. Effectivement.

Revenons à l'écriture et plus particulièrement à votre travail d'artisan, de confectionneur de romans. Quelle est votre théorie de l'écriture?

H. A. La littérature est avant tout pour moi un travail de conception, avant d'être un travail d'écriture. Cette phase de production, de conception d'un livre me paraît aussi importante que la phase d'exécution, de rédaction. L'écriture, l'exécution, c'est la phase seconde, elle est un tout petit peu secondaire. La première phase est pour moi l'étape la plus lente et la plus obsédante. Puis soudain, j'élabore un plan et j'écris. Une fois le livre bien en plan, bien organisé dans ma tête, je peux y aller, je suis sûr de mon coup. Mais il faut d'abord que je pense comment agencer tel événement, tel épisode avec tel autre, dans quel contexte, pour ensuite arriver aux petits détails de la conception, c'est-à-dire attribuer les noms aux personnages, aux lieux, etc. Le détail du plan, c'est tout l'enchaînement des séquences, donc c'est très détaillé. J'ai toujours fonctionné ainsi: une phase de conception et de maturation, puis un plan qui se consolide et qui. consolide les divers éléments, une sorte de combinatoire qui agence tout l'ensemble.

A. G. Ceci au moment du plan? 
H. A. Oui, car les modifications survenues pendant l'écriture sont en général rares et peu importantes. Dans le cas de l'Antiphonaire, j'ai retiré des éléments du plan que j'ai publiés dans Point de fuite.

A. G. Vous avez parlé d'une combinatoire?

H. A. C'est un jeu de combinaison, au sens où je joue avec plusieurs clés et plusieurs registres, où j'essaie d'agencer au mieux les séquences et de jouer sur toutes sortes d'effets de correspondance, surtout de synchronisation.

A. G. Et au troisième temps, au moment d'écriture, de la mise en chantier du plan, qu'est-ce qui se passe?

H. A. C'est un moment où j'écris en toute sécurité, bien rapidement, bien facilement. Je sais qu'il y a des écrivains qui partent avec une idée confuse de ce qu'ils vont faire par la suite et du coup il se crée une tension fantastique dans leur écriture, une tension due au fait qu'ils ne savent pas trop ce qu'ils vont écrire dans dix pages ou dans deux chapitres. Alors ça doit être absolument aberrant comme situation. On commence sans trop savoir où on va. Ça ne m'est jamais arrivé d'avancer dans le noir comme ça.

A. G. Mais il vous est déjà arrivé de perdre un plan?

H. A. Oui. J'avais perdu le plan de Trou de mémoire et cela m'a obligé à une performance absolument épuisante. Lorsque j'ai perdu le plan, j'étais déjà bien avancé dans la rédaction et je dois dire qu'une fois le livre bien avancé, j'oublie le reste du plan, je ne me casse pas la tête, je sais que tout est pensé. Au moment où j'ai perdu le plan j'avais malheureusement oublié la fin; c'était trop compliqué. J'ai dû en reformuler un. J'en ai refait deux ou trois je crois, c'est-à-dire des plans qui changeaient, des versions possibles, avec des variantes.

A. G. Le roman aurait sarement été différent si vous n'aviez pas perdu le plan initial?

H. A. Oui, oui sûrement. Mais attention. La boucle se serait bouclée de la même façon, c'est plutôt les agencements des séquences qui auraient été différents.

A. G. Je suis un peu étonnée, comme lectrice, d'apprendre que tout est tellement planifié. Lisant Prochain Épisode, $j$ 'avais l'impression de lire un livre qui se concevait et s'écrivait au fur et à mesure: I'histoire en Suisse affectant le narrateur en prison, le démoralisant et l'état de démoralisation jouant sur les actes du héros en Suisse. L'effet de lecture du jeu de miroir d'une histoire sur lautre fait qu'on ne peut imaginer que l'écriture puisse être tellement planifiée à l'avance.

H. A. Admettons que je sois un autre et que j'essaie de comprendre Prochain Épisode, je pourrais chercher à comprendre la genèse du livre et être mystifié par certaines constantes. Par exemple, les deux niveaux de l'histoire sont tout de suite établis dès le début. C'est donc dire que vraisemblablement, l'un des niveaux ne pourra sauter en 
chemin. De même il y a deux ou trois plaques tournantes, en terme spatial, comme le château d'Echandens, qui sont établies bien solidement avec beaucoup de références. Ce sont des espèces de charnières. Alors on ne pourrait pas concevoir que j'aie commencé à écrire, par improvisation, et que j'aie fabriqué les deux niveaux et que je m'y sois tenu comme on se cramponne à une nouvelle en chemin. Mais que je me sois cramponné à des trucs comme le château d'Echandens, bourré de meubles et de significations, là ce serait incompréhensible. On pourrait, en cherchant à reconstituer la genèse du livre, arriver à constater qu'il y a, au contraire, des choses inexplicables autrement que par une construction d'après un plan.

A. G. Ce que je voulais dire: dans l'effet de démoralisation d'une histoire a l'autre...

H. A. C'est compensatoire tout le temps.

A. G. Et c'était voulu dans le plan?

H. A. Oui, c'est compensatoire. C'est l'histoire d'un statişme infini, celui qui meurt sur place, qui crève, qui s'écrase, qui n'a rien et qui pour finir projette tout dans une histoire qui se déroule ailleurs, dans un espace démesuré, libre. Donc, il y a un effet compensatoire. C'est un peu comme une fugue en musique, avec le contrepoint. Ce serait un langage contrapuntique.

A. G. Ce qui est intéressant, c'est que pour vous il y a vraiment trois étapes séparées, mais ce qui fait le succès de vos livres, c'est qu'elles soient tellement bien intégrées, comme dans Prochain Épisode par exemple, où on a l'impression de lire un livre qui s'écrit à chaque mot et que l'aventure d'écriture est une aventure qui comporte beaucoup de risques pour le narrateur.

H. A. C'était une aventure de fait, bien sincèrement, au niveau de l'aventure ontologique. J'avais le temps d'approfondir tout ce que j'écrivais...

A. G. Oui, vous étiez en prison en ce moment-là.

H. A. Là, j'étais sûr de l'écrire. Donc, il y avait une intensité d'écriture qu'on ne retrouve pas dans les autres livres.

A. G. Avant de quitter cet intéressant problème de l'écriture, nous pourrions nous attarder sur le rapport écrivain-lecteur. Vous avez déjà dit dans une interview que vous aviez eu un plaisir fou en écrivant Trou de mémoire en sachant que le lecteur, le pauvre lecteur, aurait beaucoup de difficultés à vous suivre. Cela veut donc dire que le lecteur est important pour vous.

H. A. C'est un fait. Je n'ai jamais pensé que j'écrivais pour moi, j'écris pour le lecteur.

A. G. Il est en train de lire à mesure que vous écrivez...

H. A. Jusqu'à présent, j'ai toujours imaginé le lecteur au-dessus de mon épaule, en train de me déchiffrer pendant que j'écris. Du coup ça me 
donne un stimulant particulier. En tout cas je ne peux pas me souvenir d'une seule fois où ça ne m'a pas dérangé.

A. G. Et cela peut avoir modifié l'écriture?

H. A. Tout le temps. C'est un processus continuel de réflexivité. Cela modifie mes opérations mentales, mes opérations imaginaires.

A. G. Est-ce que cela peut aller jusqu'à vous faire modifier volontairement des situations ou des scènes?

H. A. Dans Trou de mémoire oui, dans l'Antiphonaire aussi. En fait je me décharge, dans l'écriture ou dans mes livres, d'une certaine partie de mon agressivité; je deviens agressif contre le lecteur tout en me réjouissant qu'il soit éventuellement là en train de me lire et du coup, une fois que je l'ai bien attrapé dans la lecture, là je le piège, je lui rends la lecture quasiment impossible ou à tout le moins difficile.

A. G. C'est un rapport sado-masochiste?

H. A. J'accepte bien volontiers. Je me venge du lecteur, je l'admets. C'est une relation bien ambivalente. Je me réjouis de sa lecture mais je suis méchant pour lui à l'instant même où je suis conscient qu'il me réjouit de sa lecture.

A. G. I/ représente quoi le lecteur pour être une cible à ce point?

H. A. C'est une question terrible que vous me posez là. Le lecteur pose sans doute le problème de l'altérité dans toute son amplitude. L'autre, c'est d'abord un être dépourvu d'individuation, de différenciation sexuelle ou autre. C'est indifférencié.

A. G. Mais pourquoi être si méchant?

H. A. Je vous le dis, je deviens lâche un peu devant mon agressivité foncière et je me réjouis de lui rendre la lecture difficile. Je me suis même réjoui de ce que certains lecteurs aient décroché de Trou de mémoire tellement ils étaient abasourdis ou ahuris. C'est assez pernicieux, pervers comme attitude.

A. G. Cela m'étonne que vous soyez logique à ce point car je comprends le jeu de l'auteur qui sent le lecteur et se voit dans un rapport dialectique avec lui mais pas au point de se réjouir du lecteur en colère qui abandonne le livre, avec l'impression de se faire jouer, de se faire «avoir».

H. A. Non. En général, il s'agit de réactions de lecteurs ahuris, déconcertés. Pas de lecteurs en colère. Ceux qui m'en ont fait part en tout cas étaient des lecteurs qui avaient l'impression que j'avais coupé les ponts. Bref, ils ne se reconnaissaient plus dans cet écheveau.

A. G. Ça c'est le lecteur poli. Mais il y a une sorte de lecteur qui n'accepte pas de se sentir dévalorisé.

H. A. Mais au contraire, il devrait se sentir valorisé ce cher lecteur parce que justement j'imagine que c'est parce que j'ai beaucoup d'estime 
pour lui que je joue avec lui et au même niveau que lui. Je considère qu'il est capable de me déchiffrer, de me décoder et même de voir des choses que je n'ai pas vues. Bref, de jouer ce jeu.

A. G. Une dernière question, concernant votre rapport à l'écriture, comment vous situez-vous face au joual?

H. A. Là-dessus, j'ai pris une position bien nette. J'ai déjà condamné le joual parce que le joual confine à une sorte d'incommunication. Cela ne mène à rien. Toutefois je suis bien conscient que je suis moi-même influencé, je suis incapable d'écrire sereinement ou doucement la langue française en bonne et due forme et j'ai trouvé qu'il y avait dans mes livres, des manifestations d'un pseudo-joual. Par exemple, le latin dans l'Antiphonaire, les barbarismes scientifiques dans Trou de mémoire. Mais je ne veux pas écrire le joual, car c'est se condamner d'avance à écrire pour rien, pour personne.

A. G. Vous voulez dire que c'est un dialecte qui ne permet pas de communiquer avec l'extérieur?

H. A. Voilà.

A. G. Mais les Québécois entre eux?

H. A. Oui mais ça ne m'intéresse pas parce que c'est le cas du dialecte liégeois en 1880. II y a eu une surproduction de livres dans ce dialecte et maintenant c'est fini, plus personne ne les lit, même pas les Liégeois. Et. c'est à peu près ce qui va se passer pour le joual, le type de joual pratiqué dans le Cassé de Renaud par exemple. Ça va durer vingt ans et après ce sera périmé, démodé. Comme le joual est une transcription d'un parler, le parler va évoluer et du coup ce sera l'incommunication avec les Québécois. Je comprends qu'on utilise le joual à la télévision ou à la radio parce que c'est une langue de communication orale qui sert hic et nunc. Mais si on se contente de transcrire le joual, ça ne va pas, parce qu'il y a des modes d'expressions, d'idiotismes; un parler ça roule, c'est un échange continuel. Mais la littérature c'est avant tout un travail de conception et non la transcription d'un parler. Le joual, ça va pour les émissions populaires qui ne peuvent être gardées pour l'immortalité.

A. G. Mais n'y a-t-il pas une contradiction entre le fait que dans vos romans le pays est le fondement, la condition de possibilité des histoires et le refus d'en assumer les particularités du dialecte?

H. A. J'admets fort bien qu'il y a une contradiction entre le fait que je ne veuille pas du joual, que je refuse ce moyen de communication, qui est un moyen d'incommunication, et le fait que j'aie désiré manifester mon enracinement dans le pays ici. J'admets la contradiction.

A. G. Je ne crois tout de même pas que dans vingt ans on ne lira plus le Cassé.

H. A. Ce sera une pièce de musée. Mais je voudrais faire une distinction avec Michel Tremblay. On ne peut qualifier la langue parlée dans ses 
pièces, de joual. C'est une langue dynamique, québécoise, à la limite peut-être du dialecte, du joual, mais c'est un français bourré d'idiotismes québécois extrêmement truculents. C'est tout autre chose. N'importe quel Français peut comprendre. Pour moi, Michel Tremblay n'écrit pas en joual. Il écrit en français mais fait parler ses personnages comme on parle dans la rue ici.

A. G. Quelle est la différence alors?

H. A. La différence, c'est que le joual est une entreprise de destruction de la langue comme moyen de communication et chez Renaud c'était bien concerté. A certains moments, il voulait presque démolir la langue traditionnelle et il est allé trop loin dans cette entreprise-là et je crois qu'il s'en est aperçu. C'est comme dans les poèmes de Claude Gauvreau, les Boucliers mégalomanes, ça peut être formidable, ça peut avoir du succès pendant un certain temps, mais c'est de l'incommunication pure et simple. C'est du lettrisme.

A. G. Mais dans un article vous disiez qu'on ne pouvait plus aujourd'hui écrire d'après les règles de la belle langue française et qu'il fallait faire éclater la syntaxe.

H. A. C'est pour cela qu'il faut l'élargir et que je suis d'accord avec Tremblay. C'est un exemple dynamique. II a appris la langue française comme nous à l'école et il l'a fait péter en l'air en l'écrivant. II l'a enracinée ici. Comme c'est du théâtre, il faut que les gens parlent en se donnant la réplique et c'est formidable.

\section{LE RAPPORT ÉCRIVAIN-PERSONNAGES}

A. G. Abordons maintenant, si vous le voulez bien, les romans et leurs personnages. Une chose $\mathrm{m}^{\prime}$ 'a frappée dans vos romans comme étant une constance de votre thématique, c'est une situation d'echec et souvent même d'impuissance. Échec dans les relations entre les personnages et à l'intérieur des couples. N'y a-t-il pas là un état de fait qui aurait quelque chose a voir avec le pays, toile de fond de vos deux premiers romans?

H. A. Je me permettrais ici une seule analogie de fait: je pourrais imaginer que la relation qui n'a pas marché entre un homme et une femme, c'est, analogiquement, une relation du personnage-narrateur avec la société, le monde dans lequel il vit.

A. G. II y aurait une relation entre l'individu et le pays mais aussi une présence du pays comme trame de fond, comme condition de possibilité ou, dans le cas présent, comme condition d'impossibilité. Quand, dans Point de fuite, vous écrivez: «le dominé vit un roman écrit d'avance» (p. 52), c'est que l'absence de solution est déjà inscrite au départ, puisqu'on appartient à une histoire qui n'est pas encore faite et peutêtre même dans laquelle il n'y a pas encore les éléments pour qu'elle se fasse, pour qu'elle soit déjà écrite. Elle est toujours à écrire. 
H. A. Si je me limite à Prochain Épisode, je suis d'accord avec vous. Le pays est un pays rêvé mais quasiment impossible, désespérément possible. Il est souhaité, il est rêvé mais il y a une situation de rupture entre le personnage-narrateur et le pays.

A. G. Le pays est souhaité dans ce qu'il pourrait être mais il est là aussi, d'une certaine façon, et c'est dans cette mesure qu'il produit l'échec. II est là, il habite le personnage d'une présence quasi obsédante. Quand le narrateur dit a la femme qu'il aime: «tu m'es sol natal», on voit que la seule relation interpersonnelle du roman est vécue à travers le pays. Or le pays, c'est, entre autres, Montcalm qui agonise, ce sont les Patriotes qui n'ont pu faire face a une victoire possible.

H. A. Vous venez de commettre un super lapsus que je trouve extraordinaire: c'est la mort du général Wolfe.

A. G. Je sais, mais Wolfe, c'est le double renversé de Montcalm...

H. A. Oui, mais je trouve ça bon car c'est la Mort du général Wolfe que je connaissais comme tableau. Dans la mesure où l'individu vit son rapport au pays, cela ne marche pas et c'est l'histoire d'une relation brisée, d'une rupture. Mais la femme n'est pas perçue ou imaginée à travers le pays, c'est plus compliqué. Elle véhicule des reflets, des valeurs du pays. Si bien qu'elle est associée au pays. Mais elle n'est pas un prisme.

A. G. Dans Prochain Épisode, j'ai vu deux femmes. La femme de ce que j'appelle la première narration qui représente le pays dans ce qu'il a de plus lyrique, de plus désirable et d'émouvant. C'est la femme des ballades dans le bout de Papineauville, à travers laquelle il découvre l'amour. Mais il y a l'autre femme, K, la femme mystérieuse et très probablement traîtresse. Et à ce moment-là, c'est une autre lecture du pays qu'il faut faire et cela nous ramène à l'échec, à l'échec du couple aussi par le fait même. À ce sujet, j'ai remarqué qu'il n'y a jamais d'espoir dans vos romans.

H. A. Je comprends ce que vous dites mais moi je ne peux pas ressentir les choses comme cela. Au contraire, je vois beaucoup de relations positives en ce qui concerne le couple; même la relation dramatique entre RR et $P$. X. Magnant finit par être positive dans la mesure où à la fin tout se raccorde en elle et, enceinte, elle veut que son enfant s'appelle Magnant, puis il y a une harmonisation finale et très positive.

A.G. Ce qui est positif c'est peut-être comment $R R$ vit la chose à la fin mais ce n'est pas la relation de couple parce qu'elle perd les deux hommes qu'elle aime.

H. A. Oui.

A. G. Donc le couple n'est pas possible.

H. A. Je reconnais qu'on pourrait arriver à cette conclusion. 
A. G. Et dans l'Antiphonaire, c'est la même chose. Toutes les relations de couples sont brisées.

H. A. C'est bien méchant. Toutes les relations sont désintégrées. Toutes les relations institutionnelles disons.

A. G. Pas seulement.

H. A. Les autres aussi, c'est vrai.

A. G. Vous qui vous vous connaissez et savez à partir de quoi vous écrivez, êtes-vous étonné par le fait que je sois surtout frappée par le côté échec, impuissance, suicide, névrose? Trouvez-vous cela exagéré?

H. A. Oui, quand vous arrivez au mot impuissance. Même pour Prochain Épisode, je n'arrive pas à comprendre qu'on puisse voir le personnagenarrateur uniquement comme quelqu'un qui a échoué ou comme un impuissant. Car je me place dans la peau du lecteur et c'est quelqu'un qui attire le lecteur dans une série d'aventures ou de situations intellectuelles plus ou moins complexes ou confuses et qui, à cet égard, est dynamique. Donc son impuissance est relative, car pour moi la puissance ou l'impuissance d'un personnage relève d'abord du phénomène littéraire puisqu'il s'agit d'un livre. Je trouve le personnage dynamique car non seulement il se dépense follement dans des courses à travers la Suisse mais en plus il attire le lecteur dans cette poursuite folle et qui semble déboucher sur le néant. Mais malgré tout il y a des moments où ça s'arrête et il y a des fascinations qui l'amènent à découvrir la Mort du général Wolfe.

A. G. Je dirais deux choses. D'une part, vous avez bien raison d'insister sur le côté dynamique mais cela je le vois au niveau du signifiant, de la forme du livre et de sa structure sous forme d'une poursuite, de cette course folle dont vous parliez, course non seulement autour du lac Léman mais à l'intérieur des chapitres et même entre les paragraphes et les phrases. Mais non pas au niveau du thème, du signifié. D'ailleurs, vous avez fait allusion aux moments d'arrêts, de souvenirs et, là encore, cette capacité de recourir à l'imaginaire et j'ajouterais imaginaire de colonisé...

H. A. Oui, oui, je suis d'accord avec cela.

A. G. C'est sarement positif d'avoir un imaginaire aussi fort mais par ailleurs j'ai remarqué qu'à chaque fois qu'il se met à penser, à revivre des situations, c'est toujours au moment où cela fait bifurquer l'action, quand il doit poser un acte, par exemple dans la forêt, il est face à son ennemi, il doit le tuer et brusquement, pirouette, on repart dans l'imaginaire. II s'agit bien alors de moments-retardements. Alors ces passages, on les vit à deux niveaux: comme incapacité a faire face à l'acte à poser et en même temps comme richesse intérieure du personnage.

H. A. Je suis d'accord avec vous sur l'incapacité du personnage à faire face aux situations ou tout simplement sur sa procrastination, le fait 
qu'il remette toujours à un autre moment l'acte qu'il avait projeté de faire. Il est procrastiné, il se procrastine, il s'autoprocrastine indéfiniment. C'est un signe peut-être d'impuissance mais aussi de plasticité dans la vie des êtres. Bien sûr, s'ils finissent par se retrouver à soixantequinze ans toujours en état de procrastination, il s'agit alors d'échecs complets. II faut donc que la vie soit moins plastique, si je puis dire, que peut l'être l'univers fictif. Si on considère le personnage comme une figure immobile, le personnage-narrateur est vraiment impuissant, mais si on le considère dans un mouvement, il est plastique et tellement qu'il se “désidentifie» carrément, et c'est plutôt cela qui me frappe après quelques années de recul. II part avec une identité, il n'en change pas mais il cherche des attributs à son identité. Et il les cherche de manière confuse ou aberrante et puis il ne les trouve pas. Bref, il ne réussit pas à trouver son identité, tout en réussissant à agir fictivement. II est en quelque sorte ou bien en voie de «désidentification", de désintégration totale ou bien en voie de «désidentification" qui débouchera sur la découverte d'une identité profonde et extrêmement importante.

A. G. Toujours en dehors du livre!

H. A. Dans ce cas-là oui, pas de doute.

A. G. Dans les trois je crois.

H. A. Là, vous me troublez un peu. Je ne comprends pas pour l'Antiphonaire. L'identité, elle est là, étalée, exposée. Elle n'est pas remise en question. Elle est au contraire poussée à bout, à l'extrême.

A. G. Ou alors c'est une identité qui est bien affirmée, bien claire au début mais qui se désintègre.

H. A. Oui, le personnage se désintègre mais ne se "désidentifie" pas. Le narrateur de Prochain Épisode a une identité floue tandis que Christine a une identité extrêmement nette, monstrueuse. Elle en prend conscience, elle le vit. Tandis que dans Prochain Épisode le narrateur dit à un moment donné: à ce rythme-là je vais me désidentifier. De fait, c'était ça le processus.

A. G. Et pour aborder maintenant Trou de mémoire, que diriez-vous si c'était RR qui avait écrit le livre?

H. A. C'est une possibilité que je ne mets pas de côté parce que les deux personnages que je retiens le plus dans Trou de mémoire, c'est RR et $P$. X. M. Et celui qui a rebondi plus tard, c'est RR qui s'est réincarnée dans Christine, à sa façon, du moins c'est une réincarnation. Mais je n'ai jamais fait se réincarner $P$. X. M., quoique la possibilité reste là en page 95 de Point de fuite lorsque je dis que son suicide est un pseudosuicide, c'était fait pour cacher sa disparition à la police, pour lui permettre d'œuvrer dans la clandestinité.

A. G. Pour revenir un peu en arrière, au moment de Prochain Épisode, le narrateur à la fin du roman n'a pu poser son acte et tuer $H$. de Heutz. 
Puis on entre dans Trou de mémoire et l'acte est déjà posé, Joan est morte. Mais Prochain Épisode annonçait ce geste en disant qu'un jour ce qui comptera ce sera de frapper, frapper à bout portant, frapper dans le dos s'il le faut. Et avec Trou de mémoire le geste est effectivement accompli. Mais je me demande si le personnage y a gagné quelque chose car le geste, il ne peut l'assumer et le livre, le journal qu'il écrit, c'est un peu jeter "de la poudre de mots plein les yeux" comme on dit si bien dans Prochain Épisode. Même si P. X. M. semble plus consistant et a des traits moins flous que le narrateur de Prochain Épisode, sont-ils en réalité tellement différents: l'un n'a pu poser l'acte, l'autre ne peut l'assumer?

H. A. Là vous posez une question nette, je vais tenter d'y répondre d'une façon confuse. Voici : quand on parle de Prochain Épisode, la structure est relativement simple: on peut dire, le personnage-narrateur, la femme aimée ou $\mathrm{K}$, la relation, le couple. Mais quand on arrive dans Trou de mémoire, cela me gêne beaucoup que vous parliez de P. X. M. parce que ce n'est pas lui le sujet du livre. Ce n'est même pas lui qui inaugure le début du livre; il apparaît seulement dans la première moitié et ensuite le livre bascule dans une sorte de livre écrit de façon contestable, par d'autres, apocryphe dans certains cas.

A. G. Oui mais cet autrela c'est Charles-Edouard Mullahy, qui est $P$. $X$. Magnant.

H. A. C'est là que cela devient un peu contestable parce que le livre est fondé sur un trou, un trou véritable qui est l'écart entre ce dont ne se souvient plus RR et ce que P. X. M. a déjà fait. Et le livre tourne autour d'un cratère. Le livre contient plus d'un narrateur et le rôle des femmes là-dedans, c'est un peu confus. II y en a une, deux, peut-être trois ou quatre. C'est secondaire parce qu'il n'y a pas de personnage unique qui soit porteur d'intrigue ou de l'argument de l'intrigue. C'est un romanrelais; le premier étant couru par l'Africain, le deuxième repris par $P$. X. M., ensuite on pourrait se mettre, en affabulant, à imaginer qu'il y a des passages apocryphes dans ce qui est donné de $P$. $X$. M., parce que tout a été collectionné, colligé puis publié. Donc l'auteur du livre, l'auteur-narrateur serait celui qui a tout colligé et non celui qui a écrit le passage signé $P . X$. M.

A. G. II y a quatre narrateurs mais il y a quand même un personnage qui a tout organisé et cela dans un but bien précis.

H. A. Voilà, et celui-là, c'est souligné à certains moments, ce pourrait être CEM, d'autres fois on laisse entendre qu'il s'agit de RR et malgré tout on reste une patte en l'air et on peut croire que c'est RR ou quelqu'un d'autre.

A. G. Ce personnage-organisateur, il sème des signes de sa présence.

H. A. II n'y a pas de doute possible que c'est une mystification. Cela se veut une mystification de la part de ce personnage-organisateur. 
A. G. Et entin l'Antiphonaire. Rapidement, je l'aborderais par le biais de son écriture. Je vois une différence entre les deux premiers romans et le troisième. D'abord, dans l'Antiphonaire, il n'y a pas d'intrigue policière. Peut-être à cause de cela, l'écriture est plus calme, moins désordonnée, moins hallucinante. C'est plus facile à lire dans le sens plein du mot. Ça se suit mieux.

H. A. Oui, c'est plus facile à recevoir. C'est harmonieux.

A. G. Alors pourquoi?

H. A. Ce que je voulais faire c'était un livre qui se lise harmonieusement avec énormément de plaisir, d'aisance et de continuité. D'autre part, celui qui aurait lu tout le livre facilement, comme cela, à la fin il se trouve piégé par une lecture qui continue sur le même ton, dans la même langue et le même style et qui devient une série d'explosions, de déflagrations; malgré tout, c'est assez horrible à la fin. Je suis conscient de cela.

A. G. C'est horrible au niveau de l'histoire mais pas au niveau de la forme, de l'écriture.

H. A. Non, pas au niveau de l'écriture. C'est, je croirais, une sorte d'hypocrisie, de duplicité que j'ai vraiment concertée. J'ai vraiment voulu que ce soit harmonieux et charmant à lire. De fait, le livre a été reçu comme tel.

A. G. Alors, il y aurait une distorsion entre la forme et le fond. Dans Prochain Épisode, je trouvais un accord total entre les deux parce $q u ' i l$ y a une déconstruction du projet, de l'histoire, et au niveau de la forme, les chapitres sont plus flous. L'accord est donc plus ferme, plus serré.

H. A. C'est un fait.

A. G. De même dans Trou de mémoire, il y a un accord assez extraordinaire.

H. A. Mais dans l'Antiphonaire, il y a une dissociation qui devient assez cynique entre la forme et le fond. La forme continue à être apparemment harmonieuse et le fond se met à exploser, jusqu'à devenir une hétacombe.

\section{EN GUISE DE CONCLUSION : QUI EST L'AUTEUR?}

A. G. Hubert Aquin, pour terminer, une question bien indiscrète: qui est l'auteur de vos romans? De quels textes est-il constitué? Et ici je me réfere à nouveau à Roland Barthes qui dit qu'un livre est un assemblage de citations de textes antérieurs, un point de rencontre de toute une série de discours qu'on a lus, entendus, vus. Quels seraient ces discours qui vous habitent?

H. A. Mais là, je ne suis toujours pas d'accord avec Roland Barthes parce qu'alors on réduirait un individu aux données de ses influences. II 
suffirait de savoir quels sont les discours reçus pour tout de suite prévoir le déroulement suivant en fonction de la personnalité, etc, etc. Je crois qu'il y a en effet une similitude des discours, une réitération historique des discours en cela que je ne peux pas inventer beaucoup, c'est vrai, mais on peut inventer la façon de vivre ou d'être et du coup, on peut vivre différemment les mots et donc habiter différemment le langage et lui infliger des distorsions importantes, lui ajouter des variantes et des acceptions nouvelles. Il est vrai que le nombre de situations dramatiques a été exploité jusqu'au bout.

A. G. Je ne crois pas qu'il faille prendre ma question et ce que dit Barthes d'une manière aussi limitative et mathématique mais dans le sens qu'il expose dans son livre $\mathrm{S} / \mathrm{Z}$ où chaque segment de texte appartient à un discours codé (sapientiel, philosophique, culturel, géographique, etc.) Par exemple dans votre Antiphonaire, il y a beaucoup de phrases qui relèvent d'un discours culturel. Ma question est: quels sont ces textes qui vous habitent en littérature, en musique, en peinture. C'est une question bien vaste, comme la vie.

H. A. En général, la culture, comme vous en parlez, ce serait un acquis et pour moi c'est plutôt une expérimentation continuelle, quotidienne. Je. me plonge presque tous les jours dans des univers bizarres, dans des lectures extrêmement recherchées, impossibles ou insensées.

A. G. Du genre de?

H. A. Depuis un an je suis dans l'Empire romain. L'année précédente je suis remonté jusqu'au $\mathrm{I}^{\ominus}$ siècle avant Jésus-Christ. En ce moment je suis dans l'Empire romain parce qu'il y a une certaine abondance de documents et je prends autant de plaisir à lire des bouquins illisibles, - il y a des citations latines et archéologiques - que des livres du xixe siècle, un peu comme quelqu'un d'autre se détend en lisant n'importe quoi ou en regardant la télévision. Pour l'Antiphonaire, je me suis remis dans tous les auteurs cités, même si je les connaissais déjà et c'est devenu très, très présent. Donc la culture pour moi c'est quelque chose que j'expérimente continuellement et que je ne considère jamais comme acquis. Maintenant les discours qui m'habitent, je ne sais pas. Je sais que j'ai des obsessions dans le sens que j'aime beaucoup des formes de productions comme les inventaires, la comptabilité, la musique comme manière de noter, comme système de notation. Je pourrais vous faire un historique des systèmes de comptabilité depuis le xixe siècle. Je m'amuse beaucoup aussi à lire des inventaires, la façon dont la société procède pour s'inventorier elle-même. Cela me passionne. Dans mes recherches sur l'Empire romain, je me suis intéressé aux frontières de cet empire, du temps de Dioclétien, d'Auguste. Je m'intéresse aussi aux tracés de fróntières.

A. G. Vous avez vraiment un esprit d'archéologue.

H. A. Je m'intéresse aussi beaucoup à des redondances dans la langue, à des réitérations de pensée. 
A. G. Dans tous ces exemples, je retrouve la combinaison: dans les inventaires, dans la notation musicale, même dans les tracés.

H. A. C'est-à-dire qu'il y a beaucoup d'énumérations et à partir de cela je peux organiser, combiner. Ce qu'il y a de plaisant dans les énumérations, c'est qu'elles donnent généralement le sentiment d'être exhaustives. Par définition, quand vous voyez un bel inventaire, c'est exhaustif: inventaire de toutes les pièces, meubles, bibelots de la maison de je ne sais trop quel seigneur, à telle époque. C'est intéressant.

A. G. I/ y a peut-être là un sentiment de plénitude?

H. A. Sentiment d'exhaustivité et aussi peut-être d'infini. Comme dans le cas des frontières de l'Empire romain, elles se sont déplacées sans arrêt, de dix ans en dix ans sur une période de six cents ans, c'est vraiment étonnant, avec des modes de bornage, de mensuration différents. C'est étrange.

A. G. C'est amusant parce que vous regardez le présent avec ce même regard que vous avez pour étudier l'Empire romain, comme un entomologiste qui regarde ses especes.

H. A. Oui. Quand je vais dans les magasins, je m'intéresse à la façon dont on dispose les objets, la façon dont on fait l'inventaire, la manière de comptabiliser. Ce sont des énumérations, des prospections énumératives et réitératives, tout le temps. C'est une manière de voir comment une société se comptabilise, s'évalue, comment elle s'inventorie. Comment évaluer le capital d'une société, le capital humain, culturel? Et comme il y en a très peu ici, alors j'ai cherché ailleurs. En plus d'avoir cherché ici car depuis l'âge de douze ans j'étais branché sur la collection Gagnon à la Bibliothèque municipale.

A. G. Alors pour vous le Québec est pauvre?

H. A. Oui, mais le Québec n'a que trois cents ans d'histoire et c'est pauvre et ce n'est la faute ni du Québec ni des États-Unis. Mais pour les États-Unis, c'est autre chose. II y a là une dimension spatiale absolument phénoménale. Ce n'est pas la profondeur temporelle, historique, ce sont plutôt les extraordinaires régionalismes, la dimension spatiale. C'est fabuleux. Les deux combinés ensemble; c'est un des pays les plus extraordinaires qu'on puisse imaginer, je crois, tandis qu'en Europe la dimension est surtout historique, plongeante dans le temps.

A. G. En musique vous devez préférer la musique abstraite à la musique classique?

H. A. J'ai bien aimé la musique de Beethoven par exemple. Mais actuellement je m'intéresse surtout aux formes musicales, la façon dont elles ont été emboîtées les unes dans les autres, la façon dont elles ont évolué à l'intérieur d'autres formes musicales, etc.

(janvier 1973) 